(C) 2017 IEEE. Personal use of this material is permitted. Permission from IEEE must be obtained for all other uses, in any current or future media, including reprinting/republishing this material for advertising or promotional purposes, creating new collective works, for resale or redistribution to servers or lists, or reuse of any copyrighted component of this work in other works.

The published article is available on http://dx.doi.org/10.1109/JSTQE.2016.2630840 


\title{
Linear and nonlinear spectroscopy by a common-path birefringent interferometer
}

\author{
Fabrizio Preda, Aurelio Oriana, Julien Réhault, Lucia Lombardi, Andrea C. Ferrari, Giulio Cerullo and \\ Dario Polli
}

\begin{abstract}
We introduce a passive common-path interferometer to replace Michelson interferometers in Fouriertransform spectroscopy. Our device exploits birefringence to introduce a highly accurate delay between two orthogonal polarization components by continuously varying the material thickness. Thanks to its inherent delay stability and reproducibility, it can be used even for short wavelengths (down to $\sim 200 \mathrm{~nm}$ ) without the need for any active control or position tracking. We first demonstrate its performances in linear spectroscopy, by implementing a spectrometer and a spectrophotometer. We then extend its use to nonlinear spectroscopy and, in combination with lock-in detection at $\mathrm{MHz}$ modulation frequencies, illustrate its application to pump-probe spectroscopy with high sensitivity $\left(\Delta T / T<3 \cdot 10^{-6}\right.$ in 1 -s integration time) and broad spectral coverage $(>500 \mathrm{~nm})$ and to broadband stimulated Raman scattering microscopy in the $\mathrm{CH}$ stretching region.
\end{abstract}

Index Terms-Fourier-transform spectroscopy, ultrafast optics, coherent Raman scattering, pump-probe.

\section{INTRODUCTION}

$\mathrm{O}$ PTICAL spectrometers can be divided in frequencydomain [1] and time-domain [2]. A frequency-domain spectrometer directly records the intensity spectrum $\widetilde{I}(\omega)=|\widetilde{E}(\omega)|^{2}$ of the optical waveform $E(t)$. This can be accomplished either sequentially, by measuring the intensity

Manuscript received September 1, 2016. This work was supported by the European Research Council (ERC) (project numbers 291198, 648615, 665635 and Hetero2D, HiGRAPHINK), EU Graphene Flagship, EPSRC Grants $\mathrm{EP} / \mathrm{K} 01711 \mathrm{X} / 1, \quad \mathrm{EP} / \mathrm{K} 017144 / 1, \quad \mathrm{EP} / \mathrm{N} 010345 / 1, \quad \mathrm{EP} / \mathrm{M} 507799 / 1$, EP/L016087/1, and the Schweizerischer Nationalfonds zur Förderung der Wissenschaftlichen Forschung (SNF) (project number PBZHP2 143444).

F. Preda, A. Oriana G. Cerullo are with the Dipartimento di Fisica, Politecnico di Milano, P.za L. Da Vinci 32, 20133 Milano (Italy) and also with the Institute of Photonics and Nanotechnologies (IFN) of the Italian National Research Council (CNR), 20133 Milano, Italy (e-mail: fabrizio.preda@polimi.it; aurelio.oriana@polimi.it; giulio.cerullo@polimi.it).

J. Réhault was with Dipartimento di Fisica, Politecnico di Milano, P.za L. da Vinci 32, 20133 Milano (Italy). He is now with the Paul Scherrer Institut, 5232 Villigen, Switzerland (e-mail: julien.rehault@psi.ch).

L. Lombardi, A.C. Ferrari are with the Cambridge Graphene Centre, University of Cambridge, 9 J.J. Thompson Avenue, Cambridge CB3 OFA, UK(11455@cam.ac.uk,acf26@eng.cam.ac.uk).

D. Polli is with the Dipartimento di Fisica, Politecnico di Milano, P.za L. da Vinci 32, 20133 Milano (Italy) and also with the Center for Nano Science and Technology at Polimi, Istituto Italiano di Tecnologia, via Giovanni Pascoli 70/3, Milan, Italy (e-mail: dario.polli@polimi.it). of different frequency components, selected by a tunable bandpass filter (such as a monochromator) or in parallel, by spatially separating the different frequencies using a dispersive optical element (such as a prism or a grating) and measuring their intensity simultaneously using a multichannel detector (such as a diode array or a charge-coupled device). Time-domain, or Fourier-transform (FT) spectrometers [2] work on a different principle: they use an interferometer that creates two time-delayed replicas of the light to be measured, $E(t)$ and $E(t-\tau)$, both of which impinge on a single-pixel detector. By measuring the overall incident energy as a function of optical path difference of the interferometer, one obtains the delay-dependent signal [2]:

$$
U(\tau)=\int_{-\infty}^{+\infty}|E(t)+E(t-\tau)|^{2} d t=U_{D C}+U_{A C}(\tau)
$$

where:

$$
\begin{gathered}
U_{D C}=2 \int_{-\infty}^{+\infty}|E(t)|^{2} d t \\
U_{A C}(\tau)=\int_{-\infty}^{+\infty} E(t) E *(t-\tau) d t+\text { c.c. }
\end{gathered}
$$

The first term (Eq. 2a) is a constant offset, not dependent on the delay $\tau$, while the second (Eq. $2 b$ ) is an interferogram which oscillates with the carrier period. By Fourier transforming this second term and recalling the WienerKintchine theorem [3], one obtains:

$$
\widetilde{U}_{A C}(\omega)=2|\widetilde{E}(\omega)|^{2}=2 \widetilde{I}(\omega)
$$

thus retrieving the intensity spectrum of the optical waveform.

FT spectrometers are widely used in many fields, such as astronomy [4], biology [5]-[7] and atmospheric sciences [8],[9], as well as for nuclear magnetic resonance [10]. They have the the following advantages: (i) they require a single element detector, which is especially important in spectral ranges where multichannel detectors are expensive or not available, such as the mid-infrared (MIR) FTIR detectors [11]; (ii) when the sensitivity is limited by the detector noise, such as in the MIR, they perform better than multichannel detectors (the so-called Felgett advantage [12]); (iii) their spectral resolution $\Delta v$, which is inversely proportional to the maximum delay $\tau_{\max }$ of the interferometer $\left(\Delta v \propto 1 / \tau_{\max }\right)$, can be easily varied according to the required measurement precision by 
adjusting the scan range; (iv) due to the lack of frequency selective elements, their optical throughput is very high, approaching unity (the so-called Jacquinot advantage [13]), which is especially important when working under low light conditions.

Typically, FT spectrometers rely on an amplitude division interferometer [14] (such as a Michelson or a Mach-Zehnder interferometer) for the generation of the time-delayed replicas. As the interferogram oscillates at the optical carrier frequency, its acquisition requires a delay precision much better than the optical period, so that any mechanical vibration of one arm of the interferometer, with a fraction of the wavelength amplitude, would spoil the interference pattern. This calls for active stabilization of the interferometer [15] or tracking with an auxiliary beam (as in commercial FTIR instruments) and makes it difficult to use FT spectrometers at shorter wavelengths, such as the near-infrared (NIR) or visible range, unless sophisticated closed-loop control systems are implemented [15].

Birefringent interferometers allow to overcome these difficulties and to achieve high passive delay stability of the generated replicas. Instead of separating the two replicas in space, as in a Michelson interferometer, a birefringent interferometer projects them onto two perpendicular polarization states, which are delayed with very high accuracy by varying the thickness of a birefringent material and then projected back to a common polarization state, in order to allow their interference on the detector. Scanning time-domain birefringent interferometers were developed for astronomic applications [16],[17], exploiting their high throughput in order to measure weak emission spectra from stars and nebulae, and were later implemented in imaging spectrometers [18]. Alternatively, static birefringent interferometers use a pair of birefringent wedges with optical axes rotated by $90^{\circ}$ sandwiched between crossed polarizers, which introduce a delay varying along the transverse spatial coordinate [19]. In this way, they produce fringes in the spatial domain, recorded by a detector array. They have the advantages of avoiding scanning elements and allowing single-shot acquisition of the interferogram, but the drawbacks of requiring a multichannel detector, and a very high sensitivity to inhomogeneities in the spatial profile of the measured beam.

We introduced a passive birefringent interferometer for FT spectroscopy in the visible/NIR [20]. It is based on a simplified version (employing only two birefringent wedges instead of four) of the Translating-Wedge-based Identical pulses eNcoding System (TWINS), formerly introduced by our group for two-dimensional electronic spectroscopy [21]. TWINS is similar to a Babinet-Soleil compensator: while the latter is typically used as a variable wave plate [22], with retardation of one or a few optical waves, the TWINS system is designed to generate pulse replicas with retardation of hundreds of optical cycles (i.e. hundreds of femtoseconds).

The advantages of TWINS are its simplicity, compactness and low cost, which make it attractive for many applications. TWINS can be used to build a compact FT spectrometer also at wavelength ranges, such as visible or UV, where pathlength stabilization in classical Michelson interferometers becomes technically too demanding. In addition, TWINS can be used in nonlinear spectroscopy for time-domain FT detection of broadband differential signals, exploiting sensitive modulation transfer schemes with high-frequency modulation and synchronous detection with lock-in amplifiers [23], [24]. This approach exploits the linearity of the FT operator, which allows one to obtain the differential spectrum as the FT of the delay-dependent demodulated signal.

Here we showcase the use of our TWINS interferometer for a variety of spectroscopic applications. Section II reviews the TWINS principle and presents the details of its experimental implementation and characterization. Section III presents several applications: a spectrophotometer to measure the absorption spectrum of a sample, a broadband pump-probe spectrometer working at $20-\mathrm{MHz}$ modulation rate and a broadband Stimulated Raman Scattering (SRS) setup. Section IV outlines future implementations of TWINS for broadband spectroscopy.

\section{TWINS DESIGN AND CHARACTERIZATION}

The principle scheme of the TWINS interferometer is shown in Fig. 1: it consists of two blocks, A and B, of the same birefringent material, with optical axes (see orange arrows in Fig. 1) perpendicular to the propagation direction and rotated by $90^{\circ}$ one with respect to the other. To allow fine tuning of its thickness, block $\mathrm{A}$ is shaped in the form of two equal wedges with small apex angle (of the order of a few degrees). For an input light beam polarized at $45^{\circ}$ with respect to the optical axes, its energy is equally distributed into two perpendicularly polarized components that collinearly propagate along the fast and slow axes of the birefringent material. The delay between these two components can be controlled by varying the thickness of block A, inserting one wedge in and out of the beam (see thick blue arrow in Fig. 1). Block B introduces a fixed delay of opposite sign with respect

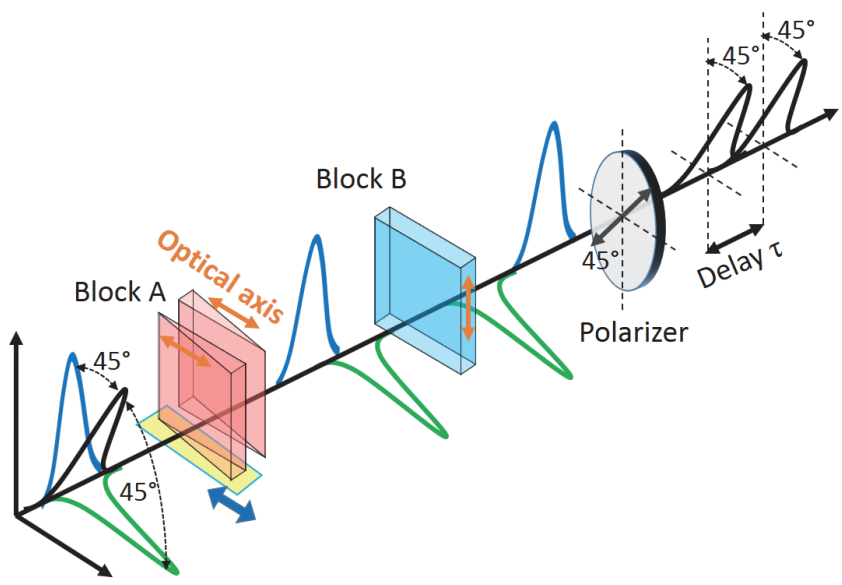

Fig. 1. Principle scheme of TWINS interferometer. The thick blue arrow indicates the direction of translation of the moving wedge. The orange arrows specify the optical axes of the birefringent optics. The black arrow marks the transmission axis of the polarizer (oriented at $45^{\circ}$ with respect to the optical axes of the birefringent optics). 
to block A, thus allowing one to achieve zero delay. Finally, after the birefringent blocks $\mathrm{A}$ and $\mathrm{B}$, a polarizer projects the orthogonally polarized components to a common polarization state, to allow their interference on a detector.

The two key characteristics of TWINS, that make it an ideal tool for FT spectroscopy, are interferometric stability and extreme delay precision ( $\sim 5$ as at $600 \mathrm{~nm}$ carrier wavelength [21]). The first property stems from its common-mode geometry: any mechanical vibration of its optical components is experienced by both light replicas, so that their relative delay is not perturbed. It can thus guarantee exceptional (better than $\lambda / 300$ in the visible [21]) path-length stability and reproducibility, with attosecond precision in the introduced delay. To understand the second property, we recall that, for a given translation $\Delta L$ of the wedge in block $\mathrm{A}$, the corresponding delay variation between the two replicas is [25]:

$$
\tau_{\text {TWINS }}=\Delta L \tan \alpha\left(\frac{1}{v_{g o}}-\frac{1}{v_{g e}}\right)=\frac{\Delta L \tan \alpha\left(n_{g o}-n_{g e}\right)}{c}
$$

where $c$ is the speed of light in vacuum, $\alpha$ is the apex angle of the wedges, $\mathrm{v}_{\mathrm{go}}\left(\mathrm{v}_{\mathrm{ge}}\right)$ and $\mathrm{n}_{\mathrm{go}}\left(\mathrm{n}_{\mathrm{ge}}\right)$ are the group velocity and the group refractive index of the ordinary (extraordinary) polarization, respectively. Equation 4 should be compared to the corresponding one for a classical Michelson interferometer [2] $\tau_{M I}=2 \Delta L / c$, where $\Delta L$ in this case represents the translation of the moving mirror. This shows that in TWINS the delay induced by a given wedge translation is demultiplied, with respect to a classical Michelson interferometer, by the so-called gear ratio:

$$
G=\tau_{M I} / \tau_{\text {TWINS }}=2 /\left[\tan \alpha\left(n_{g o}-n_{g e}\right)\right]
$$

For typical values of the geometrical and optical parameters, one has $G=30 \div 60$, thus resulting in extremely high delay accuracies. For this reason, standard mechanical delay lines with $\approx \mu \mathrm{m}$ positioning accuracy and reproducibility allow for interferometric measurements with a delay control of the order of a few tens of attoseconds [21]. We note that, due to chromatic dispersion, $\mathrm{n}_{\mathrm{go}}$ and $\mathrm{n}_{\mathrm{ge}}$ are a function of the wavelength, so that, following Eq. (4), at any given position of the translating wedge of the TWINS the delay experienced by the different spectral components is different. Calibration of the wavelength axis is performed following the procedures described in [20],[21]. This procedure also corrects for any deviation in the actual apex angle of the birefringent wedges from the desired designed one.

Considering an asymmetric interferogram with the position of the zero optical path difference close to one extreme of the scan range of the moving wedge (so as to achieve the highest spectral resolution), the maximum translation $\Delta L$ of the wedge is close to its length $L$, so that the maximum spectral resolution achievable is:

$$
\Delta v \cong\left(\tau_{\max }\right)^{-1}=\frac{c}{L \tan \alpha\left(n_{g o}-n_{g e}\right)}
$$

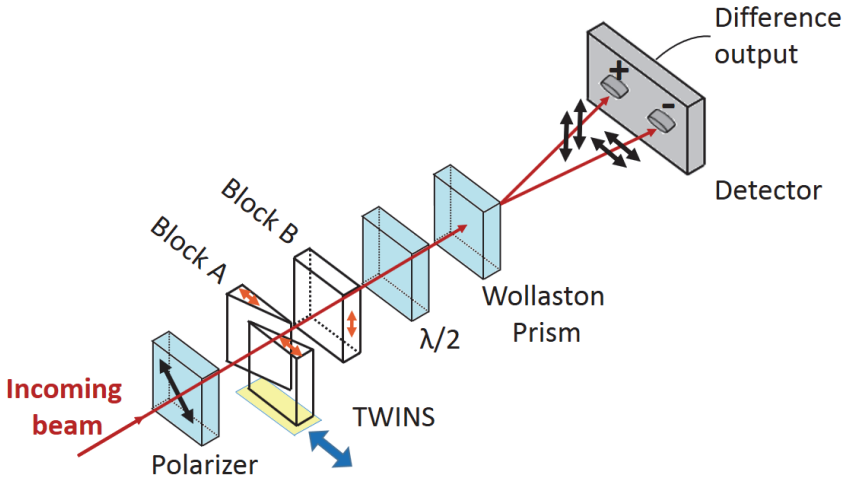

Fig. 2. TWINS spectrometer setup. The thick blue arrow indicates the direction of translation of the moving wedge. The orange arrows specify the optical axes of the birefringent optics. The black arrows mark the transmission axis of the polarizer (oriented at $45^{\circ}$ with respect to the optical axes) and the vertical/horizontal polarizations of the two beams at the output of the Wollaston prism. $\lambda / 2$ : half-wave plate.

For typical designs, $\tau_{\max }$ ranges between 1 and 3 ps, so that the spectral resolution is $10-30 \mathrm{~cm}^{-1}$, which is sufficient for most applications, such as linear absorption spectroscopy, colorimetry, pump-probe spectroscopy and Raman spectroscopy in the solid state.

Figure 2 plots the scheme of a spectrometer based on the TWINS interferometer. A polarizer at the input insures that the polarization of the input beam is at $45^{\circ}$ with respect to the optical axes of blocks A and B. After the birefringent plates, a half-wave plate rotates the polarization by $45^{\circ}$, before a Wollaston prism (WP10 from Thorlabs), so to create two orthogonally polarized beams diverging horizontally, each containing projections of the two delayed replicas. These replicas interfere at each of the two detectors of a balanced photodiode, measuring two interferograms which, due to energy conservation, are phase shifted by $\pi$ (i.e. the maximum of one channel corresponds to the minimum of the other). The differential output signal has thus zero offset and the interference fringes have double intensity with respect to those measured by a single detector. We note that the half-wave plate in Fig. 2 can also be avoided, by rotating the Wollaston prism and the photodiode by $45^{\circ}$ around the beam axis. With respect to the general scheme presented in Fig. 1, the one in Fig. 2 has the advantage of avoiding the $50 \%$ loss in energy introduced by the final polarizer, thus reaching a throughput close to unity (losses being limited only to the Fresnel reflections at the surfaces of the optical elements, that can be minimized using suitable broadband anti-reflection coatings).

We first consider Si photodiodes (PDB450A, Thorlabs, Inc.), in order to cover the visible spectral range. To characterize the spectral resolution of the instrument, we illuminate the TWINS spectrometer with a $\mathrm{He}-\mathrm{Ne}$ laser emitting a narrow line (in the $\mathrm{GHz}$ range) at $632.8 \mathrm{~nm}$. We use custom-made lithium-niobate $\left(\mathrm{LiNbO}_{3}\right)$ wedges (with birefringence $\Delta \mathrm{n}=\mathrm{n}_{\mathrm{o}}-\mathrm{n}_{\mathrm{e}}=0.1-0.08$ in the visible and $\Delta \mathrm{n}=$ 0.08-0.07 in the NIR) with $10^{\circ}$ apex angle (from Foctek Photonics Inc., Fuzhou, China). The wedge is translated to its maximum excursion (32-mm travel range) to provide the highest spectral resolution. Portions of the recorded 

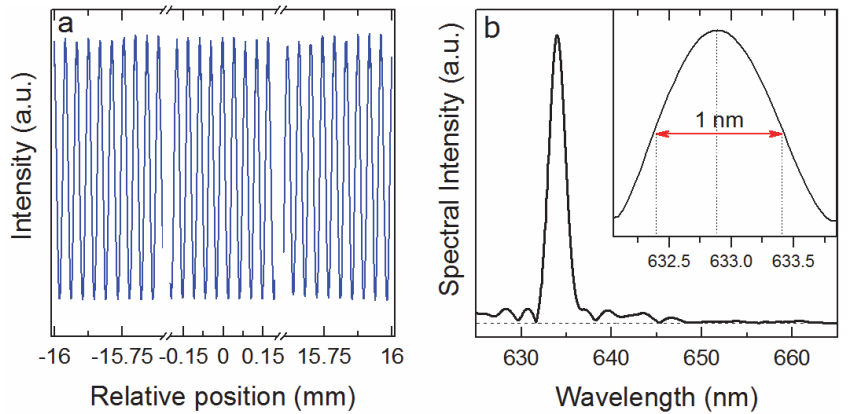

Fig. 3. (a) Interferogram measured with a He-Ne laser in a 32-mm-long scan of the moving wedge. (b) Retrieved spectrum. Inset: zoom of the spectrum, pointing out a spectral resolution of $1 \mathrm{~nm}$.

interferogram are shown in Fig. 3(a). The amplitude of the fringes remains nearly constant over the scan range, as this is shorter than the coherence length of He-Ne laser $(\approx 20 \mathrm{~cm}$ for our multi-mode He-Ne lasers). Figure 3(b) plots the corresponding spectrum, retrieved by means of a FT after apodization of the recorded interferogram with a Gaussian window. Weak residual oscillations $(<5 \%$ relative intensity) are present around the peak (within $\pm 10 \mathrm{~nm}$ ), due to a slight anharmonicity of oscillations induced by small systematic positioning errors of the translation stage. As highlighted in the inset of Fig. 3(b), the spectral resolution is $\approx 1-\mathrm{nm}$. If needed, higher spectral resolutions could be achieved: (i) using a material with higher birefringence, such as rutile $\left(\mathrm{TiO}_{2}\right)$ with $\Delta \mathrm{n}=-0.3$; (ii) increasing the scan range of the moving wedge; or (iii) widening its apex angle.

We then replace the silicon detector with an InGaAs one (PDB450C, Thorlabs, Inc.) to test the capability of our spectrometer to measure a structured NIR spectral profile. For this purpose, we use as a light source a broadband supercontinuum generated in a highly nonlinear fiber. Figure 4 plots the spectrum of the laser measured with a commercial InGaAs spectrometer (dotted red line), superimposed to the spectrum measured with the TWINS spectrometer (solid black line) after calibrating for the InGaAs responsivity. The

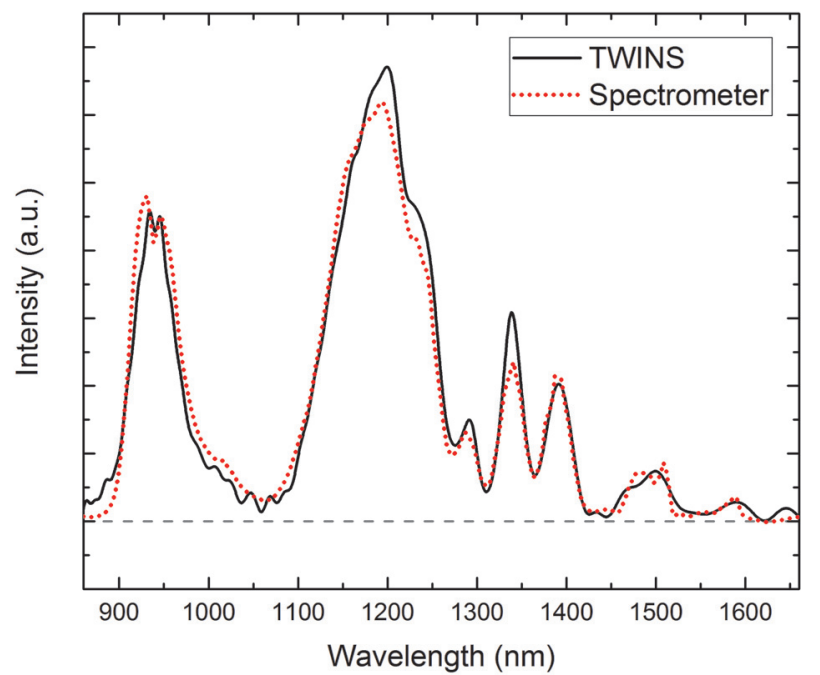

Fig. 4. Supercontinuum spectrum measured with the TWINS (black solid line) and with a standard spectrometer (dotted red line). agreement is very good and the discrepancies are mainly due to an inhomogeneous spectral content of the laser beam across its spatial profile. While the TWINS spectrometer is not equipped with an entrance slit, so that it measures the average spectrum (spatially integrated across the entire beam), the commercial InGaAs spectrometer is based on a standard imaging design, with an entrance slit, a grating and focusing optics, so that different spectra are collected across its spatial profile.

\section{TWINS APPLICATIONS}

\section{A. Application to linear spectroscopy: a spectrophotometer}

We first exploit the TWINS to implement a spectrophotometer capable of measuring absorption spectra of samples with high resolution $(\sim 1 \mathrm{~nm})$ in the visible/NIR wavelength range [20]. The experimental setup is sketched in Fig. 5. It is very similar to that in Fig. 2, the only difference being the insertion of the sample to be characterized in one of the two beams generated by the Wollaston prism. Since the interferograms generated by the two outputs of the Wollaston prism are in antiphase, their Fourier transforms differ just by a phase term, so that their power spectra are identical, and one of them can serve as a reference, allowing to record accurate absorption spectra of samples in a single measurement. Dividing the spectrum of the light transmitted by the sample by that of the reference arm, one compensates for any spectral intensity fluctuation of the incident light. We illuminate the samples with a high-power supercontinuum fiber laser (NKT Photonics model SuperK Extreme EXW-12), emitting an ultra-broadband spectrum in the 500-2300 nm range. Figure 6 shows as a gray area the measured spectrum, which extends only up to $\sim 1050 \mathrm{~nm}$ due to the responsivity of the Si detector used for these experiments. We note that similar results could also be obtained employing an incoherent source, such as a collimated light bulb typically present in UV-VIS spectrophotometers. In the latter case, the drawback of reduced brightness could be alleviated using a photomultiplier instead of a photodiode as detector. Fig. 6 shows as solid lines

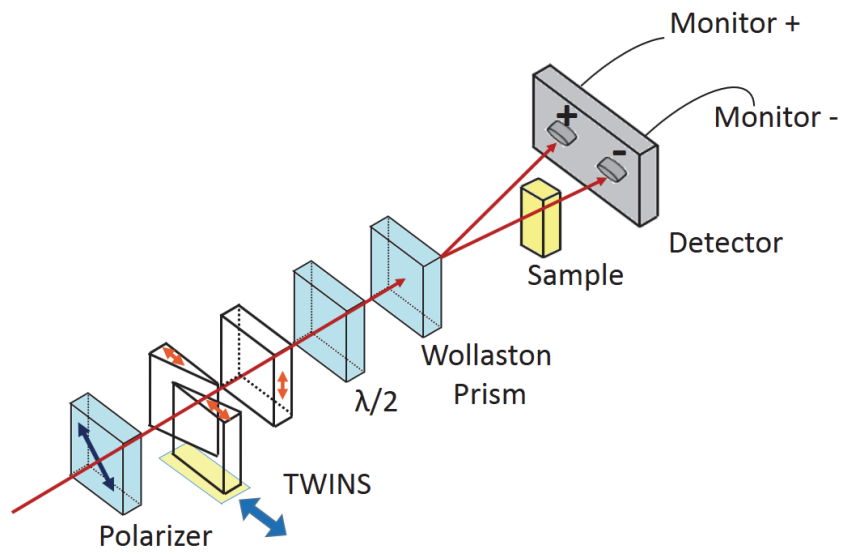

Fig. 5. Sketch of the setup of the spectrophotometer. The thin blue arrow marks the optical axis of the polarizer (oriented at $45^{\circ}$ ), the thick light-blue arrow shows the direction of movement of the wedge and the orange arrows indicate the optical axes of the birefringent optics. $\lambda / 2$ : half-wave plate. 


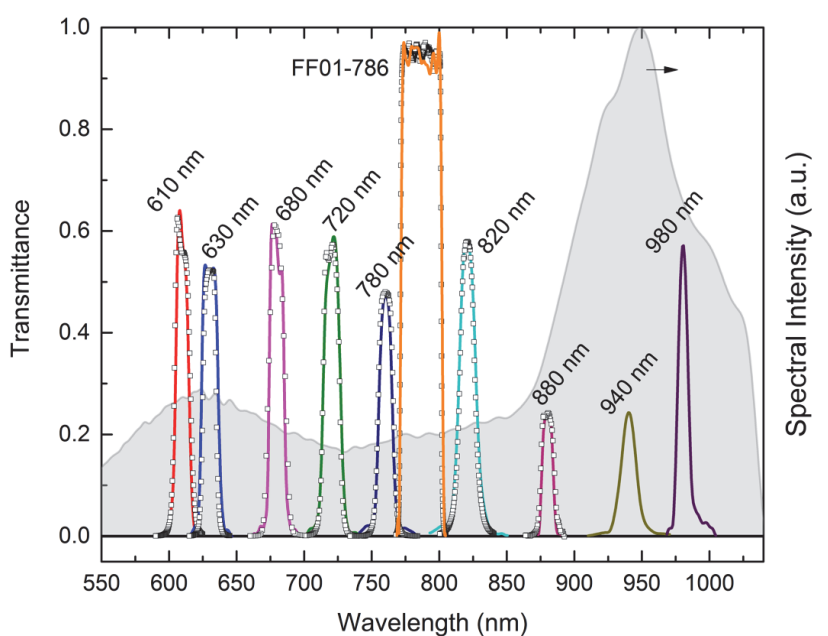

Fig. 6. Transmission spectra of interferential filters at different wavelengths and of a bandpass filter (Semrock FF01-786), measured with the TWINS (solid lines) and with a commercial spectrophotometer (squares).

the transmission spectra, measured with the TWINS spectrophotometer, of 10-nm bandwidth interferential filters (Corion series BPF10) at different wavelengths in the visible/NIR spectral region (solid lines). Fig. 6 also plots the transmission of a band-pass filter (Semrock model FF01-786). The results are in excellent agreement (both for the shape and the amplitude) with the transmission spectra measured with a standard spectrophotometer based on a monochromator (squares in Fig. 6).

\section{B. Application to nonlinear spectroscopy}

We then apply the TWINS interferometer to broadband nonlinear spectroscopy, in the pump-probe [24] and SRS [23] configurations. Since both techniques use the same experimental approach to extract the differential signal, for the sake of clarity we will explain it in detail for the pump-probe case. In a pump-probe experiment a first ultrashort light pulse, the pump, excites the sample and modifies its transmission, inducing a frequency-dependent gain or loss in a time-delayed probe pulse that contains information on the sample dynamics. Modulation transfer is typically used to sensitively detect the pump-induced transmission change of the probe: the pump is periodically switched on and off and the nonlinear interaction in the sample transfers the pump modulation to the probe.

If the probe spectrum is detected in the frequency domain with a spectrometer, the maximum refresh rates of line-scan cameras limit the modulation frequency to a few $\mathrm{kHz}$ [26][28]. On the other hand, to increase the sensitivity, one should modulate the pump at higher frequencies in the $\mathrm{MHz}$ range (ideally at exactly half the laser repetition rate), to benefit from the lower relative intensity noise of the probe beam at these high frequencies [29]. For $\mathrm{MHz}$ modulation, synchronous detection is performed with high-frequency lockin amplifiers, which however work only for a single channel, so that information on the frequency dependence of the probe transmission change is lost.

Our approach combines FT spectroscopy with highfrequency single-channel lock-in detection, enabling the use of broadband probe pulses, and thus the sensitive measurement of their spectral changes over a wide frequency range. This is possible thanks to the linearity of the FT operator, which guarantees that the difference between the FTs of two interferograms is equal to the FT of the difference of the two interferograms. A lock-in amplifier, in fact, extracts the difference of two signals, i.e. the intensity of the probe light transmitted by the sample in the presence and in the absence of the pump. Therefore, the FT of the interferogram of the demodulated lock-in signal corresponds to the difference between the probe spectra in the presence and absence of the pump.

A scheme of our setup for broadband pump-probe spectroscopy with time-domain detection is shown in Fig. 7. The modulated pump and the probe, delayed by $\tau_{\mathrm{pp}}$, are combined on the sample, after which the probe beam, either spatially or spectrally filtered, is sent to the TWINS interferometer, generating two phase-locked replicas with delay $\tau$. We note that, as the TWINS are placed after the sample, the pulse duration is not required to be kept constant, so that also in this case we can employ the simplified version of the TWINS lacking the two isotropic wedges. The energy of the replicas is measured with a photodiode, whose output is recorded by an analog to digital converter (ADC), obtaining the probe pulse interferogram $I_{p r}(\tau)$, whose FT gives the probe spectrum $\widetilde{I}_{p r}\left(\omega_{p r}\right)$. At the same time, the output of the photodiode is sent to a lock-in amplifier, whose demodulated output is the probe differential interferogram $\Delta I_{p r}\left(\tau, \tau_{p p}\right)$, giving, after FT, the differential probe spectrum $\Delta \widetilde{I}_{p r}\left(\omega_{p r}, \tau_{p p}\right)$. The differential transmission $(\Delta \mathrm{T} / \mathrm{T})$ spectrum of the probe pulse is finally computed as:

$$
\frac{\Delta T}{T}\left(\omega_{p r}, \tau_{p p}\right)=\frac{\Delta \widetilde{I}_{p r}\left(\omega_{p r}, \tau_{p p}\right)}{\widetilde{I}_{p r}\left(\omega_{p r}\right)}
$$

This approach provides a unique way to measure small spectral changes (down to a few parts in $10^{6}$ ) in broadband light pulses using a single photodetector and $\mathrm{MHz}$ modulation frequencies. In the following we will present the detailed experimental setups and results for pump-probe and SRS.

\section{B1. Broadband pump-probe spectroscopy}

The broadband pump-probe setup starts from a mode-

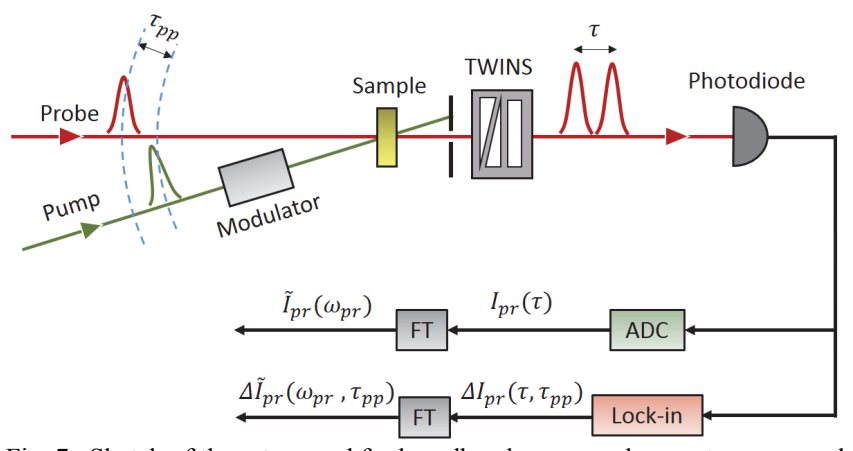

Fig. 7. Sketch of the setup used for broadband pump-probe spectroscopy and SRS. 
locked Er-doped fiber oscillator (FemtoFiber Pro from Toptica Photonics) generating 70 -fs pulses at $1.55-\mu \mathrm{m}$ central wavelength and $40-\mathrm{MHz}$ repetition rate. The oscillator output seeds two synchronized Erbium-doped fiber amplifiers (EDFAs) each of which boosts the average output power to $350 \mathrm{~mW}$. The first EDFA output is frequency doubled in a $\beta$ barium borate crystal to generate 100 -fs pump pulses at 780 $\mathrm{nm}$, while the second output is sent to a highly nonlinear fiber (HNLF), which produces a broadband supercontinuum spanning the 950-1450 $\mathrm{nm}$ range [30], used as a probe. The pump beam is sent to an acousto-optic modulator (Gooch and Housego model AOMO 3200) synchronized with the laser clock and working at 20-MHz. A motorized delay line finely adjusts the pump-probe delay with $\sim 1 \mathrm{fs}$ accuracy and reproducibility. Pump and probe beams are non-collinearly focused on the same area on the sample at a small relative angle $\left(\approx 5^{\circ}\right)$. After the sample, the probe/Stokes beam is sent to the TWINS interferometer followed by a Wollaston prism and a balanced InGaAs detector (Thorlabs PDB450C), to obtain an interferogram with double amplitude and zero offset (see Fig. 2). For these and the following experiments, we use custom-made alpha barium borate $(\alpha-\mathrm{BBO})$ wedges (from Foctek Photonics Inc., Fuzhou, China) with apex angle $\alpha=7^{\circ}$ and $25-\mathrm{mm}$ lateral size, that guarantee a spectral resolution down to $\sim 5 \mathrm{~nm}$ in the NIR. According to Fig. 7, the detector output is connected to an ADC card and to a high-frequency lock-in amplifier (HF2LI from Zurich Instruments) to measure simultaneously the probe spectrum $\widetilde{I}_{p r}\left(\omega_{p r}\right)$ and the differential probe spectrum $\Delta \widetilde{I}_{p r}\left(\omega_{p r}, \tau_{p p}\right)$. The time constant of the lock-in amplifier is typically set to a value $\sim 100$ times smaller than the time required for the wedge to scan a fringe of the interferogram, of the order of $100 \mu \mathrm{s}$.

To test the performance of the pump-probe setup, we measure a multi-layer graphene sample prepared by liquid phase exfoliation of graphite, similar to those used as a saturable absorber in mode-locked lasers [31]. The sample is produced by vacuum filtration of $500 \mu \mathrm{L}$ of ink, diluted in 1 $\mathrm{ml}$ of deionized water (DIW). The film is then transferred on a $100-\mu \mathrm{m}$ glass coverslip. The protocol to obtain the ink is as follows [32],[33]: $120 \mathrm{mg}$ of graphite flakes (Sigma-Aldrich) are dispersed in $10 \mathrm{~mL}$ of DIW with $90 \mathrm{mg}$ sodium deoxycholate, then placed in an ultrasonic bath for $9 \mathrm{~h}$ and subsequently ultracentrifuged using a TH-641 swinging bucket rotor in a Sorvall WX-100 ultracentrifuge at $10 \mathrm{krpm}$ (17 000g) for $1 \mathrm{~h}$. After ultracentrifugation, the top $70 \%$ of the dispersion is extracted by pipetting and then vacuum filtered via $100 \mathrm{~nm}$ pore-size filters (Millipore nitrocellulose filter membranes). In order to remove the surfactant, the film is rinsed by vacuum filtration of $20 \mathrm{ml} \mathrm{DIW}$. The transfer is done by applying pressure and heat $\left(90^{\circ} \mathrm{C}\right.$, to improve adhesion) overnight $(10 \mathrm{~h})$, followed by dissolution of the filter in acetone. To remove acetone residuals, the sample is then rinsed in isopropyl alcohol and in DIW baths.

The two-dimensional map of the differential transmission signal $\Delta \mathrm{T} / \mathrm{T}\left(\lambda_{\mathrm{pr}}, \tau_{\mathrm{pp}}\right)$ as a function of wavelength $\lambda_{\mathrm{pr}}$ and probe

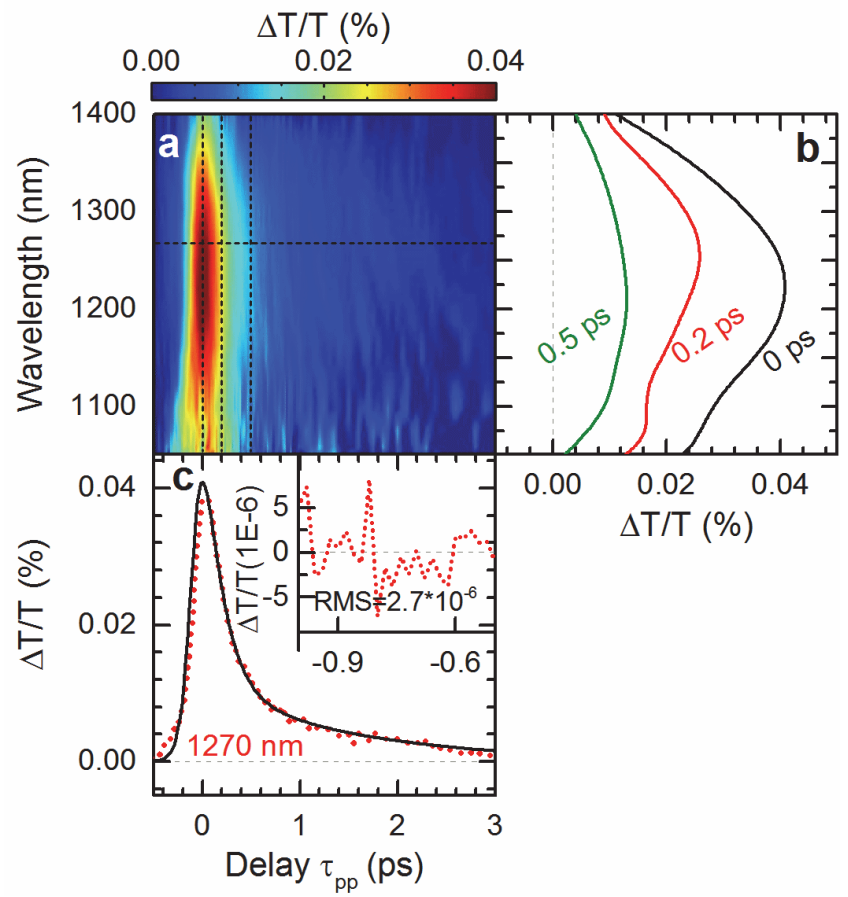

Fig. 8. (a) Two-dimensional $\Delta \mathrm{T} / \mathrm{T}(\lambda, \tau)$ map for a graphite sample prepared by liquid phase exfoliation. (b) $\Delta \mathrm{T} / \mathrm{T}$ spectra at selected probe delays; (c) $\Delta \mathrm{T} / \mathrm{T}$ dynamics at $1270-\mathrm{nm}$ probe wavelength (red circles) together with a biexponential fit (black solid line). Inset: zoom of the signal for negative delays.

delay $\tau_{\mathrm{pp}}$ is shown in Fig. 8(a). For this experiment the translating wedge of TWINS is moved at $6 \mathrm{~mm} / \mathrm{s}$ constant speed on a $3-\mathrm{mm}$ travel range, resulting in a $\approx 50 \mathrm{~nm}$ spectral resolution and an acquisition time of 0.5 -s per $\Delta \mathrm{T} / \mathrm{T}$ spectrum. A close-up of the signal at negative time delays for $\lambda=1270$ $\mathrm{nm}$ (inset of Fig. 8(c)) highlights the high signal-to-noise ratio of our $\Delta \mathrm{T} / \mathrm{T}$ spectra (rms fluctuations of the signal as low as $\Delta \mathrm{T} / \mathrm{T}=2.7 \times 10^{-6}$, after averaging 3 scans, corresponding to 1.5 seconds total integration time).

We observe for all probe wavelengths a positive signal, due to ground-state photo-bleaching, which displays a biexponential decay (see black solid line in Fig. 8(c)), with a fast time constant $\left(\tau_{1}=180 \mathrm{fs}\right)$ followed by a slower one $\left(\tau_{2}=\right.$ $1.5 \mathrm{ps})$. We assign the faster decay to the interaction with optical phonons, and a the slower one to that with acoustic phonons. The first process corresponds to our $\tau_{1}$ and the second to our $\tau_{2}$, consistent with what previously reported for graphite [34], or for single layer graphene prepared by mechanical exfoliation [35], chemical vapor deposition [36],[37], or multilayer graphene on $\mathrm{SiC}$ [38].

\section{B2. Broadband Stimulated Raman Scattering Microscopy}

Coherent Raman scattering (CRS) [39] is a powerful tool for label-free identification of molecules in cells and tissues, based on their intrinsic vibrational response, with growing applications in biomedical optics [40]. Among CRS techniques, SRS is emerging as the one of choice due to advantages, such as the linear concentration dependence of the signal, and the lack of non-resonant background [41]. In SRS the sample is illuminated by two synchronized pulses, the 
pump (at frequency $\omega_{\mathrm{p}}$ ) and the Stokes (at frequency $\omega_{\mathrm{s}}$ ). When the pump-Stokes frequency detuning matches a characteristic vibrational frequency $\Omega$ of the system under study, i.e. $\omega_{p^{-}} \omega_{\mathrm{S}}=\Omega$, then the two pulses interact via the resonant vibrational third-order nonlinearity, resulting in amplification of the Stokes pulse (Stimulated Raman Gain, SRG) and simultaneous attenuation of the pump pulse (Stimulated Raman Loss). SRS is thus technically similar to pump-probe, since one has to detect a tiny (typically a part in $10^{4}-10^{6}$ ) differential signal (the SRG or SRL) sitting on a large background (the Stokes or pump intensity).

In broadband SRS one records the full SRG/SRL spectrum, delivering an information which is equivalent to that of spontaneous Raman, but potentially with a much shorter acquisition time. Broadband SRS is technically very demanding, as the SRG/SRL signal is typically very small $\left(\Delta \mathrm{T} / \mathrm{T} \approx 10^{-4}\right.$ or less) under conditions relevant for biomedical applications [42]. Most current implementations use a serial approach, in which both pump and Stokes pulses are narrowband and the SRS spectrum is acquired by rapidly scanning their detuning across the vibrational resonances of interest [43], [44]. Alternatively, one can detect the full SRG/SRL spectrum in parallel using a spectrometer but with a low readout rate which strongly limits the sensitivity [45]. Multichannel lock-in amplifiers have been employed, but only at low modulation frequencies, resulting again in low sensitivity [46]. Much better results were obtained using a multi-channel active filter, consisting of a series of electronic band-pass filters and trans-impedance amplifiers [42], although with a limited number of pixels.

Here we demonstrate time-domain broadband SRS using FT detection. The principle of the measurement is the same as in Fig. 7, with the probe replaced by a broadband Stokes pulse which is temporally overlapped with the narrowband pump. We start from the Er:fiber laser described in the previous section, with two synchronized EDFA outputs. The first is frequency doubled in a $1-\mathrm{cm}$ long periodically poled $\mathrm{LiNbO}_{3}$ crystal, producing a narrowband $\left(\sim 15 \mathrm{~cm}^{-1}\right)$ 783-nm pump pulse with average power up to $110 \mathrm{~mW}$ and $\sim 2 \mathrm{ps}$ duration [47]. The second output is spectrally broadened in a highly non-linear fiber (HNLF), resulting in a short-wavelength dispersive wave covering the $950-1100 \mathrm{~nm}$ range, used as the broadband Stokes pulse. The corresponding pump-Stokes frequency detuning is $2000-3500 \mathrm{~cm}^{-1}$, fully covering the $\mathrm{CH}$ stretching band, relevant for many biological applications. Pump and Stokes, synchronized by a delay line, are collinearly combined by a dichroic beam splitter and focused onto the sample by a 0.75 NA objective. The pump pulse is acoustooptically modulated at $3 \mathrm{MHz}$. After the sample, the Stokes is recollimated by a second identical objective, filtered from the pump by a long-wave pass filter and sent to the TWINS interferometer. Similar to what described in the previous section and in Fig. 7, an ADC is used to record the Stokes interferogram and, by FT, the Stokes spectrum $\widetilde{I}_{S}(\omega)$, while an high-frequency lock-in amplifier is used to record the SRS

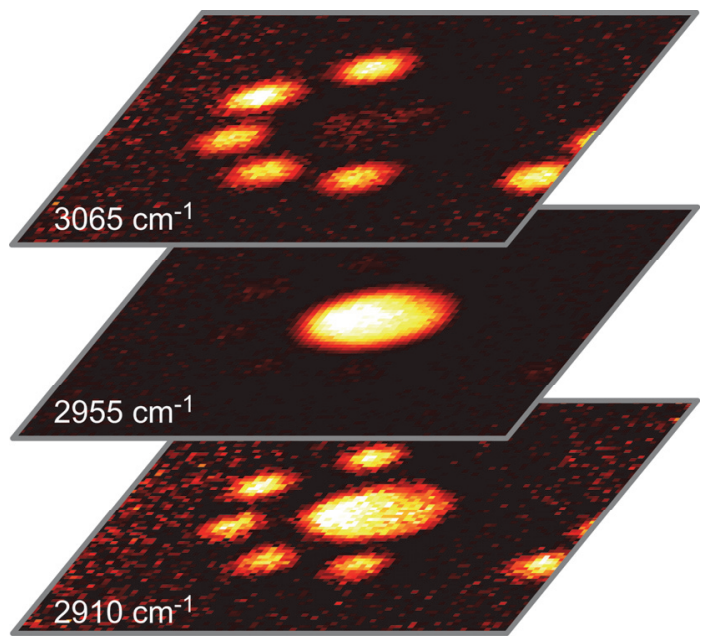

Fig. 9. Cuts of the three-dimensional SRG data-matrix measured on the PMMA/PS beads sample at specific Raman shifts, as indicated.

interferogram and, by FT, the SRS spectrum $\Delta \widetilde{I}_{S}(\omega)$. The SRG spectrum is then calculated as $\operatorname{SR} G(\omega)=\Delta \widetilde{I}_{S}(\omega) / \widetilde{I}_{S}(\omega)$. We use a $20-\mathrm{mm}$ travel range of the wedge, which corresponds to a 1-ps delay and to a frequency resolution of $\approx 30 \mathrm{~cm}^{-1}$. Given limitations in the translation speed of the wedges, the acquisition time is $\sim 1 \mathrm{~s}$.

To test our broadband SRS microscope, we image a mixture of polymethyl methacrylate (PMMA) and polystyrene (PS) spherical beads with $6-\mu \mathrm{m}$ and $3-\mu \mathrm{m}$ diameter, respectively, dispersed on a glass substrate. The spatial resolution is better than $1 \mu \mathrm{m}$. Fig. 9 reports three cuts of the measured threedimensional dataset (as a function of $x-y$ sample position and Raman shift) at three selected vibrational frequencies: 3065 $\mathrm{cm}^{-1}$, mostly in resonance with the PS, $2955 \mathrm{~cm}^{-1}$ mostly in resonance with the PMMA, and $2910 \mathrm{~cm}^{-1}$, where the two
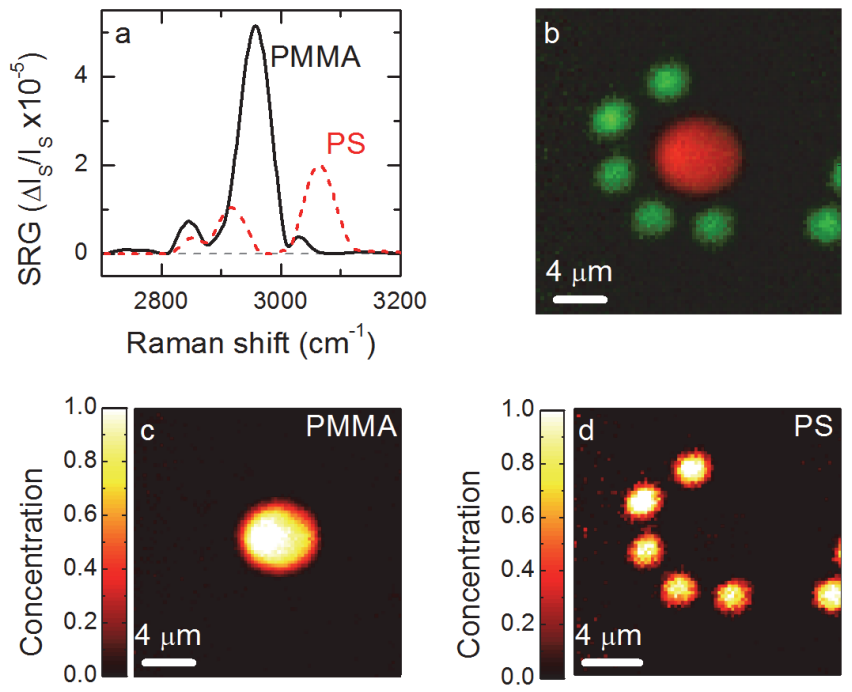

Fig. 10. Chemometric analysis of the acquired dataset. (a) SRG spectra for PMMA (solid black line) and PS (dotted red line). (b) False-color image of the sample, showing a central bead of PMMA (in red), surrounded by smaller beads of PS (in green). (c) and (d): concentrations maps of PMMA and PS. 
materials display a comparable Raman response. Fig. 10 reports the results of a chemometric analysis on the dataset, performed using the MCR-ALS Matlab routine [48], which allows one to separate the different components of the blend. We note that our broadband SRS has good sensitivity and spectral resolution, while the current acquisition speed is too slow for biomedical applications.

\section{CONCLUSIONS}

We presented the development and applications of a timedomain FT spectrometer, which measures linear and nonlinear spectra over a broad bandwidth using a single-channel detector. Our approach relies on a passive interferometer, that we called TWINS, which exploits the birefringence of an optical material to impose an arbitrary delay on two orthogonal polarization components by continuously varying the material thickness. TWINS has the following properties: (i) since both pulse replicas follow the same optical path, their delay is locked with very high stability and reproducibility (better than $\lambda / 300$ in the visible [21]) and is insensitive to mechanical fluctuations; (ii) the device demultiplies any transverse translation of the wedges, allowing extremely high delay accuracy (in the attosecond range); (iii) the device is inherently phase stable and does not require any feedback stabilization as a standard interferometer; (iv) the two pulses have pure perpendicular polarizations, which can be projected to the same direction by a polarizer. Furthermore, TWINS is a compact, rugged, and potentially low-cost device (the birefringent material, the polarizers and the translation stage could cost around a few thousand dollars).

We first reviewed the linear applications of TWINS, which allows one to measure with a single-channel detector optical spectra over a very broad wavelength range from the visible to the infrared, limited by the detector responsivity and the transparency of the birefringent material. We also applied TWINS to nonlinear spectroscopy, both in the pump-probe and the SRS configurations, demonstrating its capability to measure with high sensitivity $\left(\sim 2 \div 3 \times 10^{-6}\right)$ broadband differential transmission spectra in combination with highfrequency modulation and lock-in detection.

The delay stability of the TWINS interferometer as well as its simplicity and compactness pave the way to many future developments. The TWINS configurations we presented, using $\mathrm{LiNbO}_{3}$ and $\alpha$-BBO as birefringent materials, work over the $190 \mathrm{~nm}-5 \mu \mathrm{m}$ range. Extension to longer wavelengths, especially to the fingerprint region $(5-10 \mu \mathrm{m})$ crucial for molecular identification [11], would be enabled by the use of birefringent materials with extended MIR transparency, such as $\mathrm{Hg}_{2} \mathrm{Cl}_{2}$ (calomel) [49] and would result in simple, compact and low-cost FTIR spectrometers. The TWINS delay stability would also benefit step-scan FTIR spectroscopy, used to study structural dynamics in photoinduced reactions on the nanosecond to microsecond timescale [50]. The step-scan approach could also be used to considerably speed up the acquisition time in broadband SRS microscopy, overcoming the intrinsically limited scan speed of the wedges. Exploiting the delay stability of TWINS, one could acquire an entire SRS image for a fixed delay $\tau$ of the interferometer, and then repeat the acquisition at various delays, thus computing the FT in parallel for all pixels only at the end of the measurement. The delay accuracy of TWINS would also allow undersampling of the interferogram at a frequency which is significantly (at least 4 times) lower than the Nyquist limit [51], further reducing the acquisition time. We anticipate that, by a combination of stepscan and undersampling, it should be possible to acquire broadband SRS images in a few seconds, enabling many applications in life sciences.

\section{ACKNOWLEDGMENT}

The authors gratefully acknowledge discussions and experimental support by C. Manzoni, D. Brida, M. Marangoni, V. Kumar, F. Crisafi, G. Ciardi and D. Popa.

\section{REFERENCES}

[1] F. L. Pedrotti, L. M. Pedrotti, and L. S. Pedrotti, Introduction to Optics (3rd Edition), Pearson, 2007.

[2] S. P. Davis, M. C. Abrams, and J. W. Brault, Fourier Transform Spectrometry, Academic, 2001.

[3] A. Papoulis, Signal Analysis, McGraw-Hill Companies, 1977.

[4] P. Connes, "Astronomical Fourier spectroscopy," Annu. Rev. Astron. Astrophys., vol. 8, pp. 209-230, 1970.

[5] W. K. Surewicz, H. H. Mantsch, and D. Chapman, "Determination of protein secondary structure by Fourier transform infrared spectroscopy: A critical assessment," Biochemistry, vol. 32, pp. 389-394, 1993.

[6] Z. Movasaghi, S. Rehman, and I. U. Rehman, "Fourier transform infrared (FTIR) spectroscopy of biological tissues," Appl. Spectrosc. Rev., vol. 43, pp. 134-179, 2008.

[7] D. Helm, H. Labischinski, G. Schallehn, and D. Naumann, "Classification and identification of bacteria by Fourier transform infrared spectroscopy," J. Gen. Microbiol., vol. 137, pp. 69-79, 1991.

[8] S. Voigt, J. Orphal, and J.P. Burrows, "The temperature and pressure dependence of the absorption cross-sections of $\mathrm{NO}_{2}$ in the $250-800 \mathrm{~nm}$ region measured by Fourier-transform spectroscopy", J. Photochem. Photobiol. A, vol. 149, pp. 1-7, 2002.

[9] C. D. Boone, R. Nassar, K. A. Walker, Y. Rochon, S. D. McLeod, C. P. Rinsland, and P. F. Bernath, "Retrievals for the atmospheric chemistry experiment Fourier-transform spectrometer," Appl. Opt., vol. 44, pp. 7218-7231, 2005.

[10] R. R. Ernst and W. A. Anderson, "Application of Fourier transform spectroscopy to magnetic resonance," Rev. Sci. Instrum., vol. 37, pp. 93$102,1966$.

[11] B. C. Smith, Fundamentals of Fourier Transform Infrared Spectroscopy, CRC Press, 2011.

[12] P. B. Fellgett, "On the ultimate sensitivity and practical performance of radiation detectors," J. Opt. Soc. Am., vol. 39, pp. 970-976, 1949.

[13] P. Jacquinot, "New developments in interference spectroscopy," Rep. Prog. Phys., vol. 23, pp. 267-312, 1960.

[14] M. Born and E. Wolf, Principles of Optics. Electromagnetic Theory of Propagation, Interference and Diffraction of Light, 7th Edition. Cambridge University Press, Cambridge, 1999.

[15] A. D. Bristow, D. Karaiskaj, X. Dai, T. Zhang, C. Carlsson, K. R. Hagen, R. Jimenez, and S. T. Cundiff, "A versatile ultrastable platform for optical multidimensional Fourier-transform spectroscopy," Rev. Sci. Instrum., vol. 80, 2009, art. no. 073108.

[16] L. Mertz, “Astronomical photoelectric spectrometer," Astron. J., vol. 71, pp. 749-751, 1966.

[17] M. F. A'Hearn, F. J. Ahern, and D. M. Zipoy, "Polarization Fourier spectrometer for astronomy," Appl. Opt., vol 13, pp. 1147-1157, 1974.

[18] A. R. Harvey and D. W. Fletcher-Holmes, "Birefringent Fourier transform imaging spectrometer," Opt. Express, vol. 12, pp. 5368-5374, 2004. 
[19] M. J. Padgett and A. R. Harvey, "A static Fourier-transform spectrometer based on Wollaston prisms," Rev. Sci. Instrum., vol. 66, pp 2807-2811, 1995.

[20] A. Oriana, J. Réhault, F. Preda, D. Polli, and G. Cerullo, "Scanning Fourier transform spectrometer in the visible range based on birefringent wedges," J. Opt. Soc. Am. A, vol. 33, pp. 1415-1420, 2016.

[21] D. Brida, C. Manzoni, and G. Cerullo, "Phase-locked pulses for twodimensional spectroscopy by a birefringent delay line," Opt. Lett., vol. 37, pp. 3027-3029, 2012.

[22] K. Iizuka, Elements of Photonics, Volume 1, John Wiley \& Sons, 2002.

[23] J. Réhault, F. Crisafi, V. Kumar, G. Ciardi, M. Marangoni, G. Cerullo, and D. Polli, "Broadband stimulated Raman scattering with Fouriertransform detection," Opt. Express, vol. 23, pp. 25235-25246, 2015.

[24] F. Preda, V. Kumar, F. Crisafi, D. G. Figueroa del Valle, G. Cerullo, and D. Polli, "Broadband pump-probe spectroscopy at 20-MHz modulation frequency," Opt. Lett., vol. 41, pp. 2970-2973, 2016.

[25] J. Réhault, M. Maiuri, A. Oriana, and G. Cerullo, "Two-dimensional electronic spectroscopy with birefringent wedges," Rev. Sci. Instrum., vol. 85, 2014, art. no. 123107.

[26] U. Megerle, I. Pugliesi, C. Schriever, C. F. Sailer, and E. Riedle, "Sub50 fs broadband absorption spectroscopy with tunable excitation: putting the analysis of ultrafast molecular dynamics on solid ground," Appl. Phys. B, vol. 96, pp. 215-231, 2009.

[27] F. M. Liebel, C. Schnedermann, T. Wende, and P. Kukura, "Principles and Applications of Broadband Impulsive Vibrational Spectroscopy," $J$. Phys. Chem., vol. 119, pp. 9506-9517, 2015.

[28] F. Kanal, S. Keiber, R. Eck, and T. Brixner, "100-kHz shot-to-shot broadband data acquisition for high-repetition-rate pump-probe spectroscopy", Opt. Expr., vol 22, pp.16965- 16975, 2014.

[29] E. R. Thoen, G. Steinmeyer, P. Langlois, E. P. Ippen, G. E. Tudury, C. H. Brito Cruz, L. C. Barbosa, and C. L. Cesar, "Coherent acoustic phonons in PbTe quantum dots," Appl. Phys. Lett., vol. 73, pp. 21492151, 1998 .

[30] F. Tauser, F. Adler, and A. Leitenstorfer, "Widely tunable sub-30-fs pulses from a compact erbium-doped fiber source," Opt. Lett., vol. 29, pp. 516-518, 2004.

[31] R. Mary, G. Brown, S. J. Beecher, F. Torrisi, S. Milana, D. Popa, T. Hasan, Z. Sun, E. Lidorikis, S. Ohara, A. C. Ferrari, and A. K. Kar, "1.5 $\mathrm{GHz}$ picosecond pulse generation from a monolithic waveguide laser with a graphene-film saturable output coupler," Opt. Expr., vol. 21, pp. 7943-7950, 2013

[32] A. Fabbro, D. Scaini, V. León, E. Vázquez, G. Cellot, G. Privitera, L. Lombardi, F. Torrisi, F. Tomarchio, F. Bonaccorso, S. Bosi, A. C. Ferrari, L. Ballerini, and M. Prato, "Graphene-Based Interfaces Do Not Alter Target Nerve Cells,” ACS Nano, vol. 10, pp. 615-623, 2016.

[33] F. Bonaccorso, A. Lombardo, T. Hasan, Z. Sun, L. Colombo, A. C. Ferrari, "Production and processing of graphene and $2 \mathrm{~d}$ crystals," Mater. Today, vol. 15, pp. 564-589, 2012.

[34] M. Breusing, C. Ropers, and T. Elsaesser, "Ultrafast Carrier Dynamics in Graphite", Phys. Rev. Lett. Vol. 102, 2009, art. no. 086809.

[35] M. Breusing, S. Kuehn, T. Winzer, E. Malic, F. Milde, N. Severin, J.P. Rabe, C. Ropers, A. Knorr, and T. Elsaesser, "Ultrafast nonequilibrium carrier dynamics in a single graphene layer," Phys. Rev. B, vol. 83, 2011, art. no. 153410

[36] D. Brida, A. Tomadin, C. Manzoni, Y.J. Kim, A. Lombardo, S. Milana, R.R. Nair, K.S. Novoselov, A.C. Ferrari, G. Cerullo, and M. Polini, "Ultrafast collinear scattering and carrier multiplication in graphene," Nat. Commun., vol. 4, 2013, art. no. 1987.

[37] J. Shang, T. Yu, J. Lin, and G. G. Gurzadyan, "Ultrafast ElectronOptical Phonon Scattering and Quasiparticle Lifetime in CVD-Grown Graphene," ACS Nano, vol. 5, pp. 3278-3283, 2011.

[38] D. Sun, Z.-K. Wu, C. Divin, X. Li, C. Berger, W. A. de Heer, P. N. First, and T. B. Norris, "Ultrafast Relaxation of Excited Dirac Fermions in Epitaxial Graphene Using Optical Differential Transmission Spectroscopy," Phys. Rev. Lett., vol. 101, 2008, art. no. 157402.

[39] C. Krafft, I. W. Schie, T. Meyer, M. Schmitt, and J. Popp, "Developments in spontaneous and coherent Raman scattering microscopic imaging for biomedical applications," Chem. Soc. Rev., vol. 45, pp. 1819-1849, 2016.

[40] J. P. Pezacki, J. A. Blake, D. C. Danielson, D. C. Kennedy, R. K. Lyn, and R. Singaravelu, "Chemical contrast for imaging living systems: molecular vibrations drive CARS microscopy," Nat. Chem. Biol., vol. 7, pp. 137-145, 2011.
[41] C. W. Freudiger, W. Min, B. G. Saar, S. Lu, G. R. Holtom, C. He, J. C. Tsai, J. X. Kang, and X. S. Xie, "Label-free biomedical imaging with high sensitivity by stimulated Raman scattering microscopy," Science, vol. 322, pp. 1857-1861, 2008.

[42] C.-S. Liao, M. N. Slipchenko, P. Wang, J. Li, S.-Y. Lee, R. A. Oglesbee, and J.-X. Cheng, "Microsecond scale vibrational spectroscopic imaging by multiplex stimulated Raman scattering microscopy," Light Sci. Appl., vol. 4, 2015, art. no. e265.

[43] L. Kong, M. Ji, G. R. Holtom, D. Fu, C. W. Freudiger, and X. S. Xie, "Multicolor stimulated Raman scattering microscopy with a rapidly tunable optical parametric oscillator," Opt. Lett., vol. 38, pp. 145-147, 2013.

[44] Y. Ozeki, W. Umemura, Y. Otsuka, S. Satoh, H. Hashimoto, K. Sumimura, N. Nishizawa, K. Fukui, and K. Itoh, "High-speed molecular spectral imaging of tissue with stimulated Raman scattering," Nat. Photonics, vol. 6, pp. 845-851, 2012.

[45] E. Ploetz, S. Laimgruber, S. Berner, W. Zinth, and P. Gilch, "Femtosecond stimulated Raman microscopy," Appl. Phys. B, vol. 87, pp. 389-393, 2007.

[46] K. Seto, Y. Okuda, E. Tokunaga, and T. Kobayashi, "Multiplex stimulated Raman imaging with white probe light from a photoniccrystal fibre and with multi-wavelength balanced detection," J. Phys. D, vol. 47, 2014, art. no. 345401 .

[47] A. Gambetta, V. Kumar, G. Grancini, D. Polli, R. Ramponi, G. Cerullo, and M. Marangoni, "Fiber-format stimulated-Raman-scattering microscopy from a single laser oscillator," Opt. Lett., vol. 35, pp. 226$228,2010$.

[48] J. Jaumot, R. Gargallo, A. de Juan, and R. Tauler, "A graphical userfriendly interface for MCR-ALS: a new tool for multivariate curve resolution in MATLAB," Chemom. Intell. Lab. Syst., vol. 76, pp. 101$110,2005$.

[49] R. Maksimenka, P. Nuernberger, K. F. Lee, A. Bonvalet, J. Milkiewicz, C. Barta, M. Klima, T. Oksenhendler, P. Tournois, D. Kaplan, and M. Joffre, "Direct mid-infrared femtosecond pulse shaping with a calomel acousto-optic programmable dispersive filter," Opt. Lett., vol. 35, pp. 3565-3567, 2010.

[50] K. Ataka, T. Kottke, and J. Heberle, "Thinner, smaller, faster: IR techniques to probe the functionality of biological and biomimetic systems," Angew. Chem. Int. Ed., vol. 49, pp. 5416-5424, 2010.

[51] P. Hamm and M. T. Zanni, Concepts and Methods of $2 D$ Infrared Spectroscopy, Cambridge University, 2011.

Fabrizio Preda was born in Bergamo (Italy) in 1990. He received his Master degree in Physical Engineering from Politecnico di Milano, Milano (Italy) in

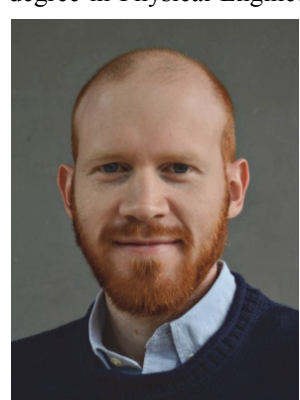
2015. The Master thesis dealt with the development of a setup for Ultrafast bidimensional electronic spectroscopy based on a birefringent interferometer, and its application to the study of photosynthetic systems as carotenoids and light-harvesting complexes.

Since 2015 , he is a research fellow involved in the "VIBRA" ERC Consolidator 2014 project in the Physics Department of Politecnico di Milano. $\mathrm{He}$ is currently working on the development of a Fourier transform-based detection system for linear and non-linear techniques, such as pump-probe spectroscopy and stimulated Raman scattering spectroscopy and microscopy.

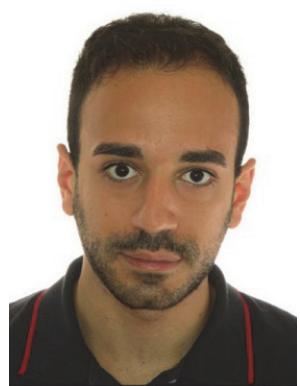

Aurelio Oriana was born in Reggio Calabria (Italy) in 1989. He received his Master degree in Physical Engineering in 2012 and his Ph.D. degree in Physics in 2016 from Politecnico di Milano, Milano (Italy). His Ph.D. thesis dealt with the development of a setup for twodimensional electronic spectroscopy based on a birefringent interferometer, and its extension to the ultraviolet range. His research interests include the generation and characterization of sub-20 fs ultraviolet pulses and their application to pump-probe spectroscopy and twodimensional electronic spectroscopy. Since 
2016, he is a research fellow involved in an ERC Advanced Grant 2012 project on two-dimensional electronic spectroscopy of biomolecules in the Physics Department of Politecnico di Milano.

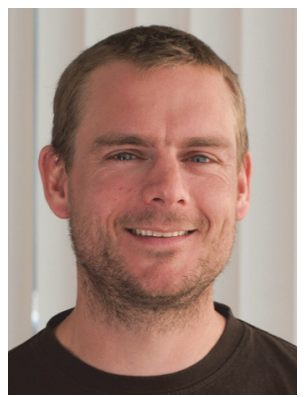

Julien Réhault was born in 1978 in France. In 2004, he started his career as an engineer for Centre national de la recherche Scientifique (CNRS). In 2008, he started a $\mathrm{PhD}$ that he received in 2012 from Zürich University. He moved for a Postdoc in Politecnico di Milano with a Swiss National Fundation grant and he is since 2015 appointed as a research scientist on the SwissFEL project in Paul Scherrer Institute. His research interests cover any type of nonlinear spectroscopy, but in particular twodimensional spectroscopy in the visible and midinfrared, with a particular emphasis on the manipulation of light polarization. He applied these techniques to the study of ultrafast photochemical and biological reactions.

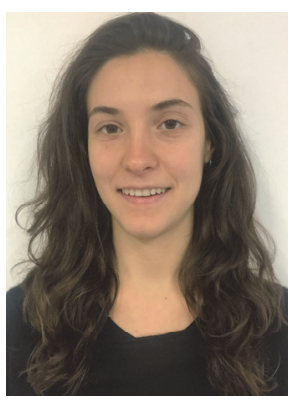

Lucia Lombardi received her Bachelor's degree in 2011 and her Master's degree in 2013, both in Materials Engineering and Nanotechnology, from Politecnico di Milano. She is currently pursuing her $\mathrm{PhD}$ degree at University of Cambridge, where she is a member of Pembroke College. Her main work focuses on formulation and characterization of graphene and related materials based inks for flexible printed (opto)electronics as well as on the development of novel $2 \mathrm{~d}$ materials based composites.

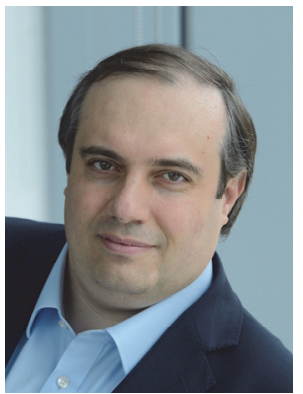

Andrea C. Ferrari is Professor of Nanotechnology at the University of Cambridge. $\mathrm{He}$ is the founding director of the Cambridge Graphene Centre (CGC) and of the EPSRC Centre for Doctoral Training (CDT) in Graphene technology. He is the chair of the Management Panel and the Science and Technology Officer of EU Graphene flagship. $\mathrm{He}$ is a triple ERC grantee, having received ERC synergy, ERC starting, and ERC proof of concept grants. He was included in the 2014, 2015, 2016 Thomson Reuters Highly Cited Researchers' Lists. He received the Royal Society Brian Mercer Award for Innovation, the Marie Curie Excellence Award, the Philip Leverhulme Prize, the Royal Society Wolfson Research Merit Award, the EU-40 Materials Prize, a Fellowship of the American Physical Society, a Fellowship of the Institute of Physics, a Fellowship of the Materials Research Society, a Fellowship of the Optical Society, a Cambridge ScD, the Charles E. Pettinos Award of the American Carbon Society, the ACS Nano Award Lectureship.

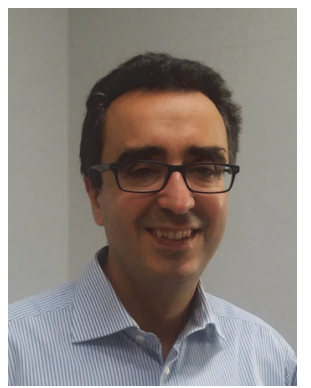

Giulio Cerullo was born in Milano (Italy) in 1965. He is a Full professor with the Physics Department, Politecnico di Milano, where he leads the Ultrafast Optical Spectroscopy laboratory. Prof. Cerullo's research activity covers a broad area known as "Ultrafast Optical Science", and concerns on the one hand pushing our capabilities to generate and manipulate ultrashort light pulses, and on the other hand using such pulses to capture the dynamics of ultrafast events in molecular and solid-state systems. Additional research topics are the applications of ultrafast lasers to nonlinear microscopy and micro/nanomachining. He has published more than 300 scientific papers on international journals, which have attracted more than 12000 citations. Since 2014 he is a Fellow of the Optical Society of America. He is the recipient of an ERC Advanced Grant (2012-2017) on two-dimensional electronic spectroscopy of biomolecules. He is responsible for the ultrafast optical spectroscopy activities in the Optoelectronic workpackage of the Graphene Flagship project. He is Program Chair of the conference Ultrafast Phenomena
2016 and General Chair of the conference CLEO/Europe 2017. He is Topical Editor of the journal Optica (Optical Society of America).

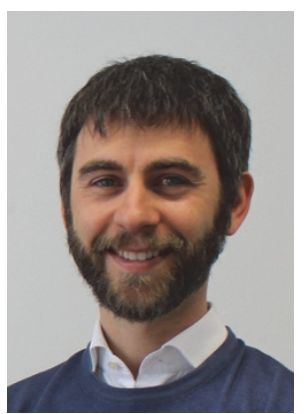

Dario Polli was born in Milan (Italy) in 1976. $\mathrm{He}$ received a double Master degree in Electronics Engineering from the Politecnico di Milano, Milano (Italy) in 2001 and in Physical Engineering from the École Centrale de Paris, Paris (France) in 2001 and the Ph.D. degree in Physics from the Politecnico di Milano in 2005. He became Associate Professor of Physics in the Physics Department, Politecnico di Milano in 2014.

His research interests include the generation and characterization of tunable femtosecond laser pulses and their application to pump-probe spectroscopy and microscopy of several samples such as carotenoids, lightharvesting systems, conjugated polymer films, bio-molecules and carbon nanotubes. Recently, he focused his attention on coherent Raman spectroscopy and microscopy, developing several techniques for stimulated Raman scattering, coherent anti-Stokes Raman scattering and Raman-induced Kerr effect.

Prof. Polli is affiliated with the Italian National Research Council (CNR) and the Center for Nanoscience and Technology of the Italian Institute of Technology (IIT). He is a member of the Optical Society of America. He was awarded with a European Research Council (ERC) Consolidator Grant in 2015 . 